\title{
Multi-variate Mutual Information for Registration
}

\author{
Jennifer L. Boes ${ }^{1}$ and Charles R. Meyer ${ }^{2}$ \\ ${ }^{1}$ The University of Michigan, Department of Radiology \\ jboes@umich.edu \\ ${ }^{2}$ The University of Michigan, Department of Radiology, \\ 3307 Kresge III Research Bldg., Ann Arbor MI 48109-0553 \\ cmeyer@umich.edu
}

\begin{abstract}
An extension of the mutual information metric to a three-variate cost function for driving the registration of a volume to pair of co-registered volumes is presented. While mutual information has typically been applied to pairs of variables, it is possible to compute multi-variate mutual information. The implementation of multi-variate mutual information is described. This metric is demonstrated using the problem of registering a deformed $\mathrm{t} 2$ slice of the visible male magnetic resonance data set to either a single $t 1$ slice or a pair of co-registered ${ }^{\circ} \mathrm{t} 1$ and proton density slices. Two-variable and three-variable metric registration results are compared. Adding the extra proton density information to the registration cost metric leads to faster optimization convergence and better final accuracy. Multi-variate mutual information has potential application in problems where the addition of more information can lead to solution convergence or improve accuracy.
\end{abstract}

\section{Introduction}

We present an extension of the mutual information metric (MI) into three variables to enable the registration of a volume with a pair of co-registered volumes. Mutual information (MI) is a metric proposed simultaneously by Collignon and Viola that works well as a cost function for registering data from different modalities [1, 2]. While mutual information is only one of a much larger set of metrics representing some kind of distance between two data sets, it has proven to work in a large number of situations. Since the early application of MI in registration, many researchers have explored its potential and limitations in this context. See for some examples [3-6]. This paper explores multi-variate computation of mutual information as a way to incorporate additional, potentially powerful information into the registration problem.

The difficulty in extending mutual information to multiple variables is the need to compute the underlying multi-dimensional probability density function (pdf). This, in general, is not an easy problem to solve. However, for this particular application, a simple brute force computation is sufficient to demonstrate the merit of the inclusion of additional evidence to solve the registration problem. The power of this extension of MI is demonstrated using simulated data based on an MRI from the visible male data. 


\section{Methods}

This paper describes a simple experiment that illustrates the usefulness of multivariate MI for registration. Existing software for MI-based registration was extended to provide for three-variate as well as two-variate MI. We ran experiments in both categories. The first category registered a slice with a known deformation back to a single slice using two-variate MI. The second category registered the same deformed slice back to a co-registered pair of slices using three-variate MI. The experiment was designed so that it would be possible to recover the exact non-affine warping applied to the deformed slice. Tests within the two categories were repeated multiple times with random starting points to provide for a robust analysis of results within and between categories.

\subsection{Optimized Registration}

The registrations were performed using the MIAMI Fuse software developed at the University of Michigan [7]. This software uses the Nelder-Mead Simplex optimizer to maximize the mutual information between a reference data set and the set to register [8]. Solution transforms can be either affine or warps, although for this experiment all solutions were warps. Parameters required are those controlling the stopping criteria of the optimizer. For this set of experiments we set the initial search bound of the optimizer to 4 voxels and set a stopping criteria of 0.5 voxel accuracy. The software runs multiple optimization cycles until the cost function changes by no more than 0.0001 for 3 out of 5 cycles. The number of cycles and corresponding MI are recorded to facilitate comparison of convergence.

\subsection{Computation of 3D MI}

The computation of mutual information for $m$ variables, more than two, requires the estimation of the $m$-variate probability density function that is the basis for MI. As $m$ grows large, this can be difficult to compute, due to problems estimating the underlying pdf of the variables. However, for small $m$ like three in this set of experiments, this problem is still tractable [9]. We have chosen to implement multivariate MI using a histogram estimation of the joint and single probability density functions. Fig. 1 shows a sample three-variate histogram. The mutual information is computed from the histogram as the sum over all gray levels trios of $P\left(g_{a}, g_{b}, g_{c}\right) \log \left\{P\left(g_{a}, g_{b}, g_{c}\right) / P\left(g_{a}\right) P\left(g_{b}\right) P\left(g_{c}\right)\right\}$. Since the $P(g)$ values are computed from the 2-variate or 3-variate histograms with $N$ samples as $H(g) / N$, this estimation depends on the selection of histogram binning. For this set of experiments, bin width for MI computation was separate and automatic for all two/three variables according to a formulation suggested by Freedman-Diaconis, $2(I Q R) n^{-1 / 3}$, where $I Q R$ is the interquartile range of the data and $n$ is the sample size [9]. This formula makes no assumptions about the shape of the probability density function. 


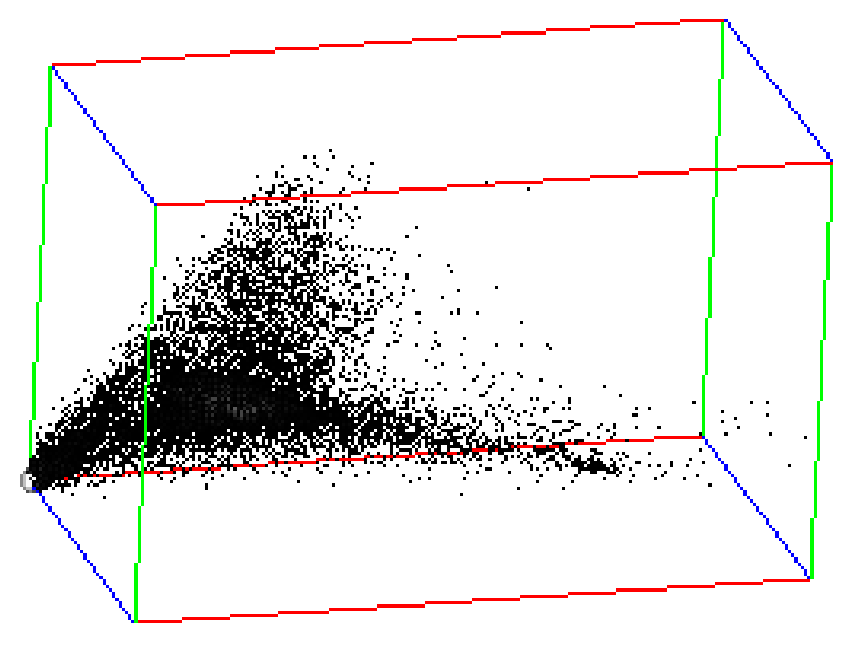

Fig. 1. Sample three-variate histogram for the estimation of the joint probability density of original slices-t1, proton density, t2-of the visible male MRI shown in Fig. 3

We can compare the two-variate and three-variate MI by graphing MI as a parameter of the transform changes. For example in Fig. 2, we use the original versions of the visible male MRIs shown in Fig. 3 and apply a thin-plate spline warp using five control points at the 4 vertices of a box and in the center of the box. The point in the center is moved in the $\mathrm{x}$ and $\mathrm{y}$ directions. The influence of this movement is shown by plotting MI in Fig. 2. Note that the additional information in the three-variate case yields a cost function with greater slope and better second derivatives near the solution.

\subsection{Simulation Data Using Visible Male MRI}

The simulation data is a trio of slices from the head of the visible male MRI data set (see http://www.nlm.nih.gov/research/visible/getting_data.html for more information on this data). Original 256x256 slices have been cropped to $167 \times 219$. Voxels are approximately $1 \mathrm{~mm} \times 1 \mathrm{~mm}$ in size. The $\mathrm{t} 1$ and proton density images were left unchanged to use as a reference data pair (Fig 3a-b) while the $\mathrm{t} 2$ image was deformed with a known transform, a 5 point (16 degree of freedom) thin-plate spline (Fig 3c). The slices remained in integer format. Experiments registered the deformed $\mathrm{t} 2$ image back to either the $\mathrm{t} 1$ or the $\mathrm{t} 1$-density pair. 


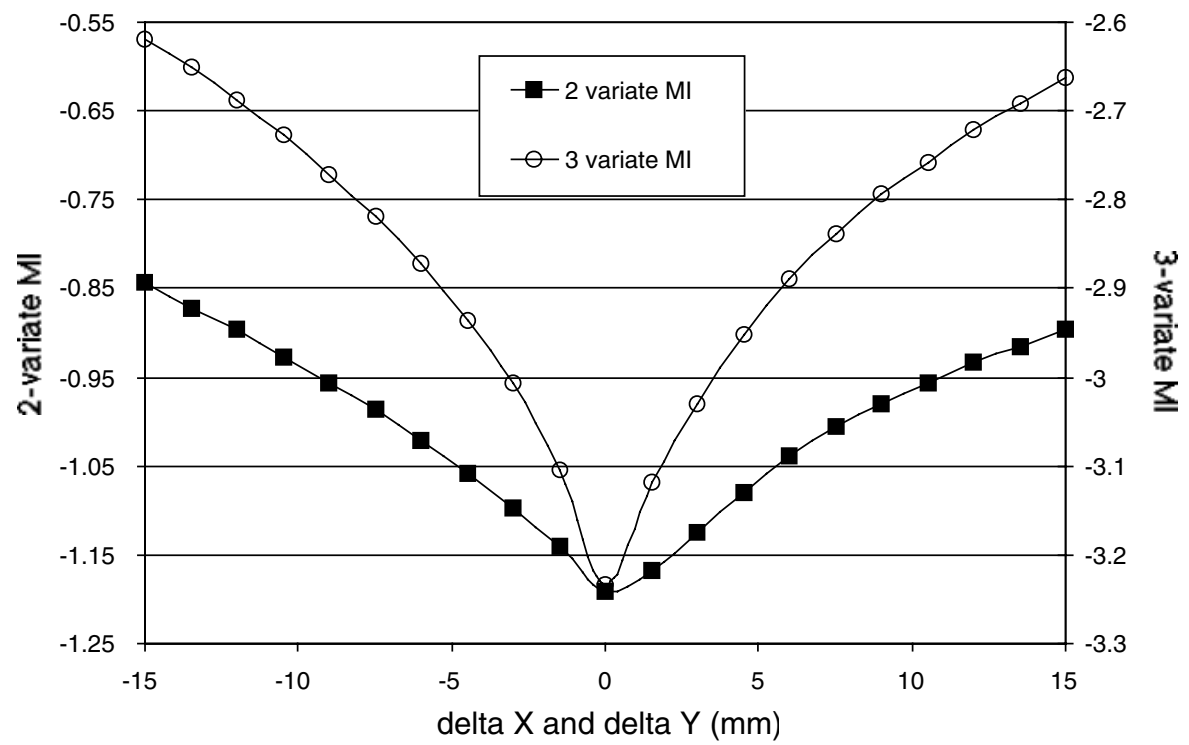

Fig. 2. Plot of two-variate and three-variate MI. Middle point of warp is being translated the same delta in $\mathrm{x}$ and $\mathrm{y}$ while outside 4 points of a box are held constant. The two graphs have been superimposed leaving vertical scales at the same units, but different range to promote visual comparison of the MI cost function progression. The lines shown are curves fit to the data points

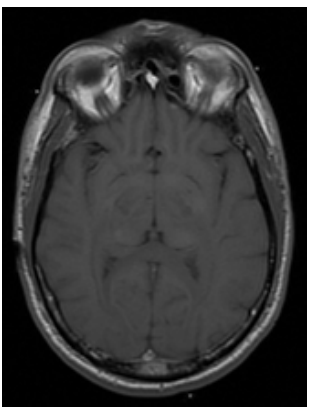

(a)

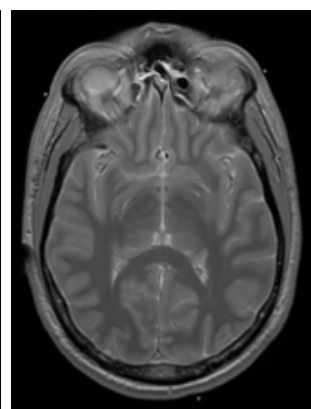

(b)

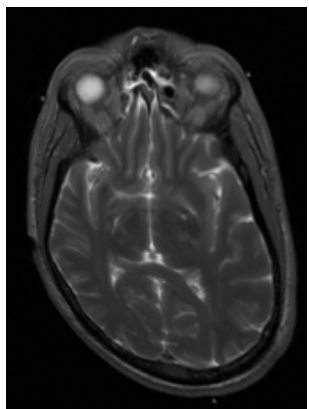

(c)

Fig. 3. Visible male MRI slice data for registration tests: (a) t1, (b) proton density, (c) warped $^{\circ} \mathrm{t} 2$

\subsection{Registration Test and Analysis}

Registrations in this experiment are based on the same feature set that was previously used to deform the image. This enables the recovery of the exact warp used to deform the slice initially. It also enables us to use the optimized locations of these points for analysis. A solution that removes the warp completely will optimize features to the 
exact original locations used to generate the warp. Good solutions yield points very near the original locations. The statistics of variation of these points from the mean and from the known solution, particularly the standard deviation $\sigma$, indicate the goodness of the solution.

\section{Results}

Twenty registrations were completed in each category, two-variate MI and threevariate MI. Results are summarized in Fig. 4 and 5 and Table 1. We expected that the three-variate case would converge more quickly and yield more accurate results for the same stopping criteria. We found that, indeed, the 3-variate MI yielded more accurate results (Fig. 4 and Table 1) with particularly good results at the feature in the center of the image, Feature 5, where the $\sigma$ of the solutions improved considerably. Interior areas often lack strong texture or features to drive accurate registration, but the new information helps to overcome this. In addition, Fig. 5 illustrates that the three-variate optimization problem converged in much fewer optimization cycles than the two-variate case.
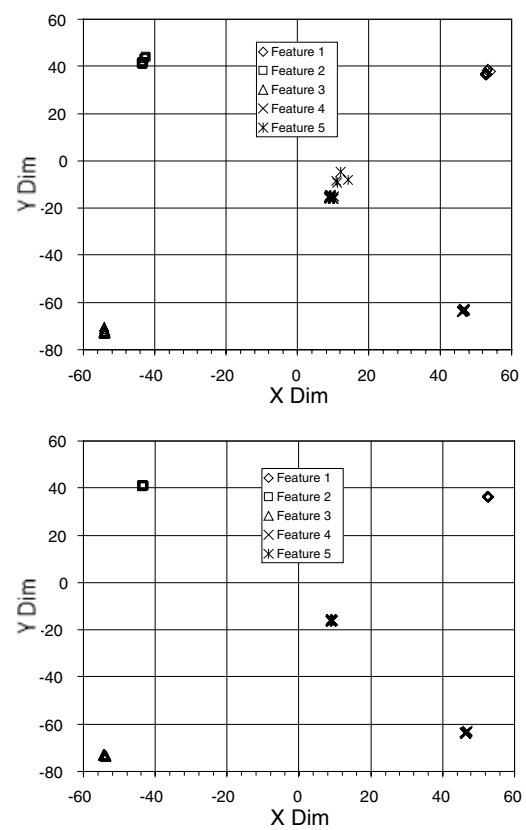

(a)

(b)

Fig. 4. Graphs of final location of feature points after optimization: (a) two-variate MI (t1 \& t2), (b) three-variate MI (t1-pd \& t2). 
Table 1. Error analysis of two variate and three variate registration solutions. Shown are the average standard deviation from the mean final location for each feature as per Fig. 4 that was used in the registration. Results are reported in millimeters

\begin{tabular}{ccc}
\hline & $\begin{array}{c}\text { MI: t1 \& t2 } \\
\sigma \text { of final loc }(\mathrm{mm})\end{array}$ & $\begin{array}{c}\text { MI: t1-pd \& t2 } \\
\sigma \text { of final loc }(\mathrm{mm})\end{array}$ \\
\hline \hline Feature 1 & 0.76 & 0.23 \\
Feature 2 & 1.06 & 0.25 \\
Feature 3 & 0.77 & 0.28 \\
Feature 4 & 0.41 & 0.31 \\
Feature 5 & 3.50 & 0.32 \\
\hline \hline Ensemble & 1.67 & 0.27 \\
\hline
\end{tabular}

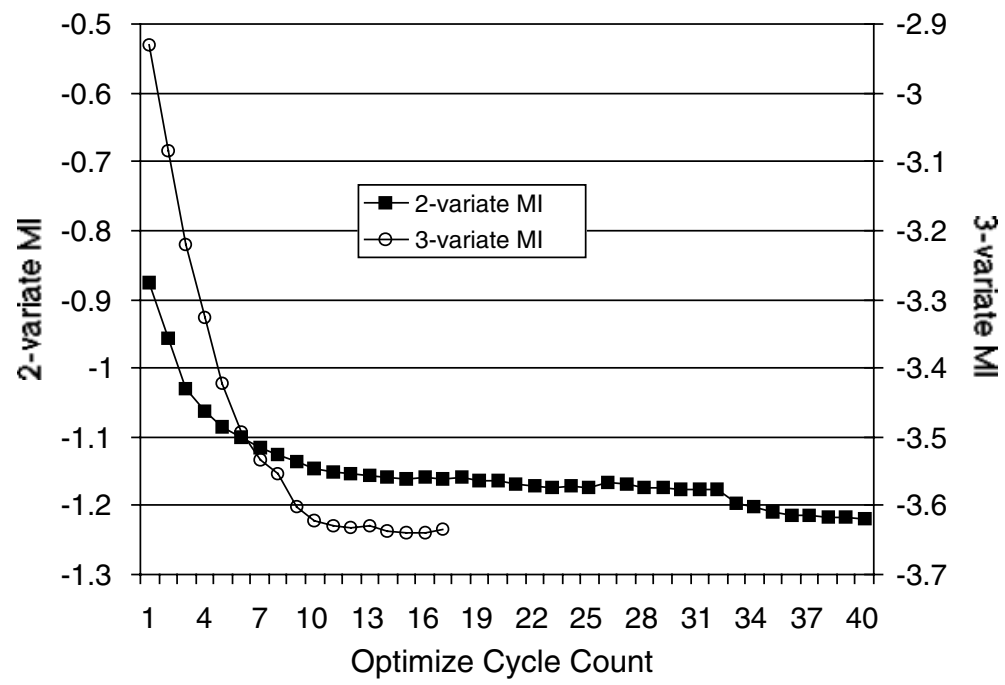

Fig. 5. Average mutual information at the conclusion of each optimization cycle. The two graphs have been superimposed leaving vertical scales at the same units, but different range to promote visual comparison of the MI progression. Numbers of cycles to solution varies with each run of the optimization algorithm. Average cycles for two-variate MI was 22 and average cycles for three-variate was 12 . 


\section{Conclusions}

We have presented a simple illustration of multi-variate MI as a cost function for driving the registration of a slice to a pair of co-registered slices. Although tests have been run on only one two-dimensional data set, the results indicate that this technique may be promising for registration problems in which additional information can either enable convergence or improve accuracy.

\section{Acknowledgements}

This work was supported in part by NIH Grants 2R01 CA59412-04A1 and 1R41 CA72351-01. Thank you to our reviewers for thoughtful feedback; our apologies for not being able to address all suggestions due to an impending move to England.

\section{References}

1. Collignon, A., Vandermeulen, D., Suetens, P., and Marchal, G. 3D multimodality medical image registration using feature space clustering. Presented at CVRMed'95, Nice, FR. In Lecture Notes in Computer Science, Vol. 905. Springer-Verlag (1995) 195-204

2. Viola, P. and Wells, W.M. Alignment by maximization of mutual information. Presented at 5th Int'l Conf on Computer Vision, Cambridge, MA. In Proceedings of the 5th Int'l Conf on Computer Vision. IEEE (1995) 16-23S

3. Kim, B., Boes, J.L., Frey, K.A., and Meyer, C.R. Mutual information for automated multimodal image warping. Presented at Visualization in Biomedical Computing, Hamburg, Germany. In Lecture Notes in Computer Science, Vol. 1131. Springer-Verlag (1996) 349354

4. Wells, W., Viola, P., Atsumi, H., Hakajima, S., and Kikinis, R.: Multimodal volume registration by maximization of mutual information. Medical Image Analysis 1:1 (1996) 3551

5. Maes, F., Collignon, A., Vandermeulen, D., Marchal, G., and Suetens, P.: Multimodality image registration by maximization of mutual information. IEEE Transactions on Medical Imaging 16:2 (1997) 187-98

6. Hill, D.L., Maurer, C.R., Jr., Studholme, C., Fitzpatrick, J.M., and Hawkes, D.J.: Correcting scaling errors in tomographic images using a nine degree of freedom registration algorithm. Journal of Computer Assisted Tomography 22:2 (1998) 317-23

7. Meyer, C.R., Boes, J.L., Kim, B., Bland, P., Zasadny, K.R., Kison, P.V., Koral, K., Frey, K.A., and Wahl, R.L.: Demonstration of accuracy and clinical versatility of mutual information for automatic multimodality image fusion using affine and thin plate spline warped geometric deformations. Medical Image Analysis 1:3 (1997) 195-206

8. Press, W.H., Flannery, B.P., Teukolsky, S.A., and Vetterling, W.T.: Numerical recipes in C: the art of scientific computing. Cambridge University Press, Cambridge (1988)

9. Izenman, A.J.: Recent developments in nonparametric density estimation. Journal of the American Statistical Society 86:413 (1991) 205-224 